\title{
Oligomerised lychee fruit-derived polyphenol attenuates cognitive impairment in senescence-accelerated mice and endoplasmic reticulum stress in neuronal cells
}

\author{
Takuya Sakurai $^{1 *}$, Kentaro Kitadate ${ }^{2}$, Hiroshi Nishioka ${ }^{2}$, Hajime Fujii ${ }^{2}$, Junetsu Ogasawara ${ }^{1}$, \\ Takako Kizaki $^{1}$, Shogo Sato ${ }^{1}$, Tomonori Fujiwara ${ }^{3}$, Kimio Akagawa ${ }^{3}$, Tetsuya Izawa ${ }^{4}$ and Hideki Ohno ${ }^{1}$ \\ ${ }^{1}$ Department of Molecular Predictive Medicine and Sport Science, School of Medicine, Kyorin University, \\ 6-20-2 Shinkawa, Mitaka, Tokyo 181-8611, Japan \\ ${ }^{2}$ Amino Up Chemical Company Limited, 363-32 Shin-ei, Kiyota-ku, Sapporo 004-0839, Japan \\ ${ }^{3}$ Department of Cell Physiology, School of Medicine, Kyorin University, 6-20-2 Shinkawa, Mitaka, Tokyo 181-8611, Japan \\ ${ }^{4}$ Faculty of Health and Sport Science, Doshisha University, 1-3, Tatara Miyakodani, Kyotanabe, Kyoto 610-0394, Japan \\ (Submitted 3 October 2012 - Final revision received 12 February 2013 - Accepted 18 February 2013 - First published online 27 March 2013)
}

\section{Abstract}

Recently, the ability of polyphenols to reduce the risk of dementia and Alzheimer's disease (AD) has attracted a great deal of interest. In the present study, we investigated the attenuating effects of oligomerised lychee fruit-derived polyphenol (OLFP, also called Oligonol) on early cognitive impairment. Male senescence-accelerated mouse prone 8 (SAMP8) mice (4 months old) were given OLFP (100 mg/kg per d) for 2 months, and then conditioned fear memory testing was conducted. Contextual fear memory, which is considered hippocampus-dependent memory, was significantly impaired in SAMP8 mice compared with non-senescence-accelerated mice. OLFP attenuated cognitive impairment in SAMP8 mice. Moreover, the results of real-time PCR analysis that followed DNA array analysis in the hippocampus revealed that, compared with SAMP8 mice, the mRNA expression of Wolfram syndrome 1 (Wfs1) was significantly higher in SAMP8 mice administered with OLFP. Wfs1 reportedly helps to protect against endoplasmic reticulum (ER) stress, which is thought to be one of the causes for AD. The expression of Wfs1 was significantly up-regulated in NG108-15 neuronal cells by the treatment with OLFP, and the up-regulation was inhibited by the treatment of the cells with a c-Jun N-terminal kinase-specific inhibitor rather than with an extracellular signal-regulated kinase inhibitor. Moreover, OLFP significantly attenuated the tunicamycin-induced expression of the ER stress marker BiP (immunoglobulin heavy chain-binding protein) in the cells. These results suggest that OLFP has an attenuating effect on early cognitive impairment in SAMP8 mice, and diminishes ER stress in neuronal cells.

Key words: Oligomerised lychee fruit-derived polyphenol: Cognitive impairment: Wolfram syndrome 1: Endoplasmic reticulum stress

Alzheimer's disease (AD) is a progressive neurodegenerative disorder pathologically characterised by the presence of senile amyloid- $\beta$ (A $\beta$ ) plaques and neurofibrillary tangles generated by $\gamma$-secretase-induced amyloid precursor protein proteolysis and tau hyperphosphorylation, respectively ${ }^{(1)}$. Recent increases in the number of patients with dementia involving $\mathrm{AD}$ are a grave public health problem, and medical costs will be commensurate ${ }^{(2)}$. Alzheimer's Disease International has recently reported that 35.6 million people worldwide live with dementia; the number has doubled every 20 years and is expected to reach an estimated 65.7 million by 2030, and $115 \cdot 4$ million by $2050^{(3)}$.
Polyphenols, such as catechin and resveratrol, are a large group of natural antioxidants that are found in fruits and vegetables, and in beverages such as tea, coffee and wine. Current studies have strongly suggested that polyphenols reduce the risk of $\mathrm{CVD}$, cancers, neurodegenerative diseases including $\mathrm{AD}$, diabetes and osteoporosis ${ }^{(4,5)}$. Indeed, there is growing evidence that intake of polyphenols is effective for $\mathrm{AD}$ risk reduction and the improvement of cognitive impairment, which is the predominant symptom of dementia involving $\mathrm{AD}^{(6,7)}$. An epidemiological study has indicated that consumption of red wine, in which polyphenols exist in abundance, is associated with a lower incidence of

Abbreviations: A $\beta$, amyloid- $\beta$; AD, Alzheimer's disease; BiP, immunoglobulin heavy chain-binding protein; ER, endoplasmic reticulum; ERK, extracellular signal-regulated kinase; JNK, c-Jun N-terminal kinase; MCI, mild cognitive impairment; OLFP, oligomerised lychee fruit-derived polyphenol; SAMP, senescence-accelerated mouse prone; SAMR, senescence-accelerated mouse resistant; Wfs1, Wolfram syndrome 1. 
dementia and $\mathrm{AD}^{(8)}$. Furthermore, improvement of cognitive performance due to the long-term (almost 5-6 months) oral intake of polyphenols, such as green tea epigallocatechin-3-gallate, has been observed in studies using an $\mathrm{AD}$ mouse model ${ }^{(9-12)}$.

The senescence-accelerated mouse, a model mouse for accelerated senescence, was established by Takeda et al. ${ }^{(13,14)}$. This senescence-accelerated mouse consists of senescenceprone (SAMP) and senescence-resistant (SAMR) inbred strains ${ }^{(13)}$. The SAMP8 strain, one of the substrains in SAMP, shows early deficits of learning and memory and early development of lesions in the brain that are similar to those seen in aged animals ${ }^{(13,14)}$. For instance, passive avoidance testing shows that the learning memory of SAMP8 mice has already declined at 2 months of age ${ }^{(15)}$. Moreover, spontaneous overproduction of $A \beta$ and hyperphosphorylated tau is found in the hippocampus of SAMP8 mice ${ }^{(16-19)}$; therefore, SAMP8 mice are proposed as a model mouse for AD.

To avoid increases in the number of patients having dementia and $\mathrm{AD}$, retarding the development of such diseases by the attenuation of early and mild cognitive impairment (MCI) is thought to be very important. Therefore, we hypothesised that the short-term administration of polyphenol could possibly be effective for the attenuation of early cognitive impairment and MCI of SAPM8 mice. In the present study, we used oligomerised lychee fruit-derived polyphenol (OLFP), which is an optimised phenolic product derived from lychee fruit-derived polyphenols and contains catechin-type monomers and low-molecular-weight oligomers $^{(20)}$. Polyphenols reportedly exert beneficial effects that reduce the risk of $\mathrm{AD}$ through the modification of intracellular molecules, such as secretase, AMP-activated protein kinase and cyclic AMP response element-binding protein, in the hippocampus ${ }^{(9,21,22)}$. OLFP has also exhibited various biological effects via the regulation of intracellular molecules. For example, OLFP enhances lipolysis in primary rat adipocytes through the activation of the extracellular signalregulated kinase (ERK) $1 / 2$ pathway, and OLFP-induced attenuation of hepatic damage in type 2 diabetic obese mice relates to the regulation of transcription factor nuclear factor- $\kappa \mathrm{B}$ expression in the liver ${ }^{(23,24)}$. Thus, we further hypothesised that OLFP target molecules must exist in the hippocampus given the attenuating effects on hippocampusdependent memory impairment in SAMP8 mice, and therefore we performed a DNA array analysis to search for OLFP target molecules in the hippocampus. Of the possible candidate factors, we chose Wolfram syndrome 1 (Wfs1), the expression of which is up-regulated in the hippocampus of SAMP8 mice by OLFP intake, because it is believed that Wfs1 impairs endoplasmic reticulum (ER) stress, which is involved in the pathology of $\mathrm{AD}^{(25-27)}$, and little is known about the effect of polyphenols on ER stress in neuronal cells. In the present study, a culture of NG108-15 neuronal cells was used to investigate whether OLFP could directly enhance the expression of $W f S 1$, and attenuate ER stress in neuronal cells by evaluating the expression of immunoglobulin heavy chain-binding protein (BiP), which is an ER stress-related marker ${ }^{(28)}$.

\section{Materials and methods}

\section{Oligomerised lychee fruit-derived polyphenol}

OLFP was obtained by oligomerising polyphenol polymers from lychee fruit-derived polyphenol using a modification of a patented technology described previously ${ }^{(20)}$. The process involves mixing proanthocyanidins with tea extract but not with L-cysteine and purifying the mixture using a column. OLFP contains $15.7 \%$ polyphenol monomer ( + -catechin and (-)-epicatechin, etc.) and $13.3 \%$ polyphenol dimer (procyanidin B2, etc.), while lychee fruit-derived polyphenol contains $6.4 \%$ polyphenol monomer and 9.9\% polyphenol dimer. The safety of OLFP as a food or dietary supplement and as a pharmaceutical additive has already been confirmed, as described previously ${ }^{(20)}$. OLFP is commercially available (Amino Up Chemical Company Limited).

\section{Animal care}

Male SAMP8 ( $n$ 12) and SAMR1 ( $n$ 6) mice (4 months old) were obtained from the Council for SAM Research via Japan SLC, Inc. Mice were housed individually in cages in a temperature-controlled room at $23^{\circ} \mathrm{C}$ under a $12 \mathrm{~h}$ light $-12 \mathrm{~h}$ dark cycle, with food and water available ad libitum. SAMP8 mice were randomly divided into two groups: a control group (SAMP8 mice: $n$ 6) and an OLFP intake group (OLFPSAMP8: $n$ 6). OLFP-SAMP8 mice were given OLFP $(100 \mathrm{mg} / \mathrm{kg}$ per $\mathrm{d}$ each) added to their drinking water for 2 months. All experiments conducted in the present study were approved by the Experimental Animal Ethics Committee of Kyorin University, School of Medicine, Mitaka.

\section{Conditioned fear memory}

Mice were tested for contextual fear conditioning and extinction using modified methods described previously by Fujiwara et $a l .{ }^{(29)}$ and Ohta et $a l .{ }^{(30)}$. For this purpose, two conditioning chambers with different shapes (context A, square; context B, triangle) were used for the conditioned fear memory test. Context A had a shock grid floor made of twenty-six stainless rods with a length of $18.0 \mathrm{~cm}(0.2 \mathrm{~cm}$ in diameter $)$ spaced $0.7 \mathrm{~cm}$ apart (centre to centre), and context $\mathrm{B}$ had stainless rods with a length from 0.8 to $15 \cdot 5 \mathrm{~cm}$. Each mouse experienced ten incidents of tone-shock (conditioned stimulus: $20 \mathrm{~dB}$ )electrical foot-shock (unconditioned stimulus: $1.0 \mathrm{~mA}, 2 \mathrm{~s}$ ) pairings in context A. After the conditioned stimulus-unconditioned stimulus pairing, mice were allowed to remain in the conditioning chamber for $30 \mathrm{~s}$ before being returned to their home cages. At approximately $24 \mathrm{~h}$ after pairing, each mouse was placed in context A to study contextual fear memory, and was scored for the freezing response for $3 \mathrm{~min}$. Moreover, to study cued fear memory, mice were returned to context $\mathrm{B}$ and scored for the freezing response to the conditioned stimulus for $57 \mathrm{~s}$ without the unconditioned stimulus.

\section{DNA array analysis}

Total RNA from the hippocampus of each mouse and neuronal cell lines was prepared using Isogen (311-02501; 
Nippon Gene). The mRNA was converted to single-stranded DNA (complementary DNA), labelled and hybridised to the Sentrix ${ }^{\circledR}$ Mouse-6 Expression BeadChip (BD-26-101; Illumina, Inc.), according to the manufacturer's instructions, and with the assistance of GP BioSciences Limited.

\section{Quantitative real-time PCR}

Complementary DNA was amplified using a TaqMan Universal PCR Master Mix (4304437; Applied Biosystems) in triplicate with a 7500 Real-Time PCR System (Applied Biosystems). PCR conditions were $50^{\circ} \mathrm{C}$ for $2 \mathrm{~min}$ and $95^{\circ} \mathrm{C}$ for $10 \mathrm{~min}$ followed by forty-five cycles of $95^{\circ} \mathrm{C}$ for $15 \mathrm{~s}$ and $60^{\circ} \mathrm{C}$ for $1 \mathrm{~min}$. Each PCR was conducted in $25 \mu \mathrm{l}$ TaqMan Gene Expression Assays for Wfs1, cerebellin 4 (Cbln4) and BiP/GRP78 (Mm00495979, Mm00558663 and Mm00517691; Applied Biosystems). TaqMan Gene Expression Assays consist of a pair of unlabelled PCR primers and a TaqMan probe with a fluorescent dye label on the $5^{\prime}$ end, and minor groove binder non-fluorescent quencher on the $3^{\prime}$ end. $18 \mathrm{~S}$ Ribosomal RNA was used as an internal control (TaqMan Ribosomal RNA Control Reagent: 4308329; Applied Biosystems).

\section{Western blot analysis}

Total protein was extracted from mouse hippocampal tissues or neuronal cells with T-PER Tissue Protein Extraction Reagent (78510; Pierce) containing protease inhibitors. Each protein sample was subjected to 10-12\% NuPAGE (NP0321BOX; Invitrogen) gradient gel electrophoresis and transferred onto a nylon membrane. The membrane was blocked using Trisbuffered saline with Tween-20 (TBS-T; 20 mm-Tris- $\mathrm{HCl}, \mathrm{pH}$ $7 \cdot 5,137 \mathrm{~mm}-\mathrm{NaCl}$ and $0 \cdot 1 \%$ Tween-20) containing 5\% nonfat dry milk, and was then probed with an anti-Wfs1 (11 558-AP; Proteintech Group), anti-BiP (SPC-107B; StressMarq Biosciences), anti-phosphorylated ERK 1/2 (sc-16982-R; Santa Cruz Biotechnology), anti-ERK1/2 (V1141; Promega), anti-phosphorylated c-Jun N-terminal kinase (JNK, 4668; Cell Signaling Technology), anti-JNK (sc-571; Santa Cruz Biotechnology) or $\beta$-actin antibody (sc-1616; Santa Cruz Biotechnology). After washing with TBS-T, the bound antibody was detected using an enhanced chemiluminescence (ECL) system (RPN2106; GE Healthcare Life Sciences). The intensities of bands from Western blot analyses were quantified using National Institute of Health Image software.

\section{Cell culture}

Neuroblastoma $\times$ glioma hybrid NG108-15 cells (ECACC 88112302) were cultured in Dulbecco's modified Eagle's medium supplemented with $10 \%$ fetal bovine serum. For neuronal differentiation, NG108-15 cells were cultured in Dulbecco's modified Eagle's medium supplemented with $1 \%$ fetal bovine serum and $1 \mathrm{~mm}-\mathrm{N}^{6}-2^{\prime}$-O-dibutyryladenosine $3^{\prime}, 5^{\prime}$-cyclic monophosphate (D0627; Sigma-Aldrich) for $4 \mathrm{~d}$. Differentiated NG108-15 cells were treated with OLFP $(2 \mu \mathrm{g} / \mathrm{ml}$ ) for $30 \mathrm{~min}, 2$ or $6 \mathrm{~h}$, and then the expression level of $W f S 1$ and the phosphorylation state of ERK or JNK were assessed. Moreover, to clarify whether the activation of ERK or JNK associates with the up-regulation of $W f s 1$ expression in NG108-15 cells by the treatment with OLFP, differentiated NG108-15 cells were pretreated with $5 \mu \mathrm{M}$-U0126 (an ERK inhibitor) or $50 \mu \mathrm{M}-\mathrm{SP} 600125$ (a JNK inhibitor) (U120 and S5567; Sigma-Aldrich) for $30 \mathrm{~min}$ before the treatment with OLFP. In addition, NG108-15 cells were cultured in the presence of tunicamycin $(0.5 \mu \mathrm{g} / \mathrm{ml}$, T7765, Sigma-Aldrich), which is an inducer of ER stress, with or without OLFP for $12 \mathrm{~h}$, and the mRNA and protein expression of BiP was examined.

\section{Data expression and statistical analysis}

Values are presented as means with their standard errors. The significance of multiple comparisons was determined using ANOVA followed by the Bonferroni post hoc test. We considered $P<0.05$ to be statistically significant. Moreover, as for the results of conditioned fear memory, the effect size (Cohen's $d$ ) was calculated using mean changes between the SAMP8 and SAMP8-OLFP mice and the pooled standard deviation. A conventional rule is to consider a Cohen's $d$ of $\geq 0.8$ or $\leq-0.8$ to be a large effect ${ }^{(31)}$.

\section{Results}

Effects of oligomerised lychee fruit-derived polyphenol intake on the conditioned fear memory of senescence-accelerated mouse prone 8 mice

In order to examine the effects of OLFP intake on the memory impairment of SAMP8 mice, we first conducted a contextual fear conditioning test, since contextual fear memory of 4month-old SAMP8 mice reportedly deteriorates ${ }^{(30)}$. The ratio of freezing responses to training in context A (contextual fear memory) of SAMP8 mice at $24 \mathrm{~h}$ after the conditioned stimulus-unconditioned stimulus pairing was significantly decreased compared with that of SAMR1 mice, but no significant difference in freezing responses was observed between the SAMR1 and OLFP-SAMP8 mice (Fig. 1(a)). On the other hand, no significant differences in cued fear memory were observed between the SAMR1 and SAMP8 mice, as reported previously $^{(30)}$, and OLFP intake did not significantly affect the cued fear memory of SAMP8 mice (Fig. 1(b)). With respect to contextual fear memories, comparing OLFP-SAMP8 mice with SAMP8 mice revealed a large effect (Cohen's $d=1.76$ ), although the size of the effect in cued fear memories was small (Cohen's $d=0 \cdot 37$ ).

\section{Effects of oligomerised lychee fruit-derived polyphenol intake on mRNA levels in the hippocampus of senescence-accelerated mouse prone 8 mice}

Contextual fear memory is considered hippocampus- and amygdala-dependent memory, whereas cued fear memory is considered amygdala-dependent memory ${ }^{(32)}$. Therefore, based on the results of Fig. 1, OLFP could rescue the impaired consolidation of contextual memory in SAMP8 mice in part through the enhancement of hippocampal function. 

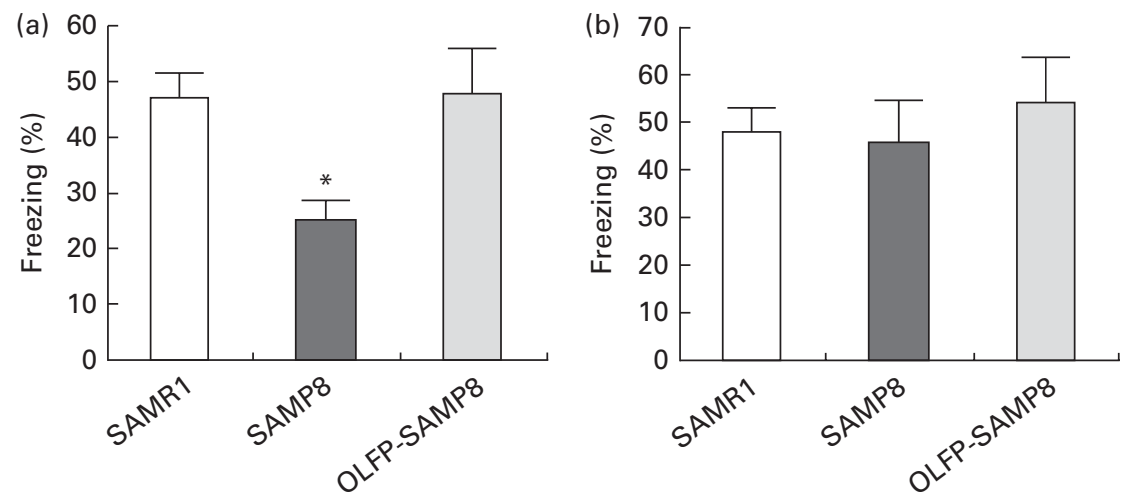

Fig. 1. Effects of oligomerised lychee fruit-derived polyphenol (OLFP) on the conditioned fear memory of senescence-accelerated mouse prone 8 (SAMP8) mice. Each mouse was trained with ten tone-shock pairings and tested for (a) contextual fear memory and (b) cued fear memory $24 \mathrm{~h}$ after training. Freezing responses to contextual and cued fear conditioning are shown. Values are means ( $n 6$ per group), with their standard errors represented by vertical bars. ${ }^{*}$ Mean value was significantly different from that of senescence-accelerated mouse resistant 1 (SAMR1) mice $(P<0.05)$.

To search for OLFP target molecules, which may relate to the OLFP-induced attenuation of cognitive impairment in the hippocampus, we next performed a DNA array analysis. The representative genes picked out in the analysis are listed in Table 1. The mRNA expression levels of $W f_{S 1}$, Cbln4, lamin B1 and matrix Gla protein in the hippocampus of OLFP-SAMP8 mice were higher than those of SAMP8 mice; on the contrary, ankyrin repeat domain 11 and guanine nucleotide-binding protein, $\alpha$ inhibiting activity polypeptide 1 were down-regulated in the hippocampus of OLFP-SAMP8 mice. The results of real-time PCR analysis revealed that the mRNA level of Cbln 4 in the hippocampus was significantly higher in OLFP-SAMP8 mice than in either SAMR1 or SAMP8 mice (Fig. 2(a), left). Moreover, compared with SAMP8 mice, the mRNA expression of $W f S 1$ was significantly increased in the hippocampus of OLFP-SAMP8 mice (Fig. 2(a), right). In addition, the expression level of the Wfs1 protein in the hippocampus of SAMP8 mice was lower than that of SAMR1 mice, and the intake of OLFP significantly enhanced the expression of the Wfs1 protein in the hippocampus of SAMP8 mice (Fig. 2(b)).

Effects of oligomerised lychee fruit-derived polyphenol on the Wolfram syndrome 1 level in NG108-15 neuronal cells

The Wfs1 protein localises primarily to the ER membrane, and is observed in the Cornet d'Ammon 1 (CA1) region of the rodent hippocampus ${ }^{(33,34)}$. Moreover, Wfs1 is suggested to have protective effects against ER stress that is involved in the pathology of $\mathrm{AD}^{(25-27)}$. Therefore, in the present study, we focused on Wfs1, and investigated whether OLFP could directly enhance the expression of Wfs1 in neuronal cells. As shown in Fig. 3, the expression of gene and protein for Wfs1 in NG108-15 cells was significantly increased by the treatment with OLFP. Polyphenols have reportedly enhanced the activation of mitogenactivated protein kinases, such as ERK and JNK, in various cell types $^{(23,35,36)}$. In the present study, the enhanced phosphorylation of ERK and JNK in NG108-15 cells was also observed due to the treatment with OLFP (Fig. 4). Furthermore, OLFPinduced enhanced expression of the Wfs1 gene and protein was inhibited by the treatment of the cells with SP600125 (a JNK inhibitor), but the treatment with U0126 (an ERK inhibitor) had no effect on the mRNA expression of Wfs1 (Fig. 5(a) and (b)). Thus, it is thought that the enhanced expression of Wfs 1 in NG108-15 cells by OLFP is mediated through the activation of JNK.

\section{Effects of oligomerised lychee fruit-derived polyphenol on tunicamycin-induced endoplasmic reticulum stress in NG108-15 neuronal cells}

We finally investigated whether OLFP diminishes ER stress by evaluating the expression of $\mathrm{BiP}$, a well-recognised marker of ER stress ${ }^{(28)}$, in neuronal cells. As shown in Fig. 6, the mRNA

Table 1. Representative genes with expression changes in the hippocampus of oligomerised lychee fruit-derived polyphenol-administered senescence-accelerated mouse prone 8 (OLFP-SAMP8) mice compared with those of SAMP8 mice*

\begin{tabular}{lccc}
\hline Genes & SAMR1 & SAMP8 & OLFP-SAMP8 \\
\hline Cerebellin 4 & 1 & 0.84 & 3.53 \\
Wolfram syndrome 1 & 1 & 0.73 & 2.34 \\
Lamin B1 & 1 & 1.33 & 3.89 \\
Matrix Gla protein & 1 & 0.26 & 0.77 \\
Ankyrin repeat domain 11 & 1 & 1.43 & 0.44 \\
Guanine nucleotide-binding protein, & 1 & 1.69 & 0.83 \\
$\quad$ & & & \\
\hline
\end{tabular}

SAMR1, senescence-accelerated mouse resistant 1.

* The expression intensities for the indicated genes are the values that are relative to the expression levels in SAMR1 mice (set to 1). 
(a)

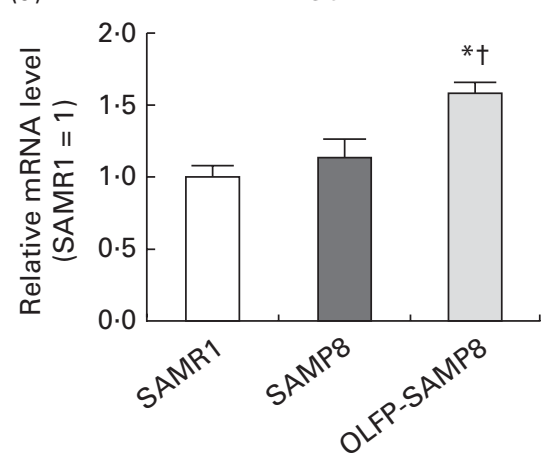

(b)

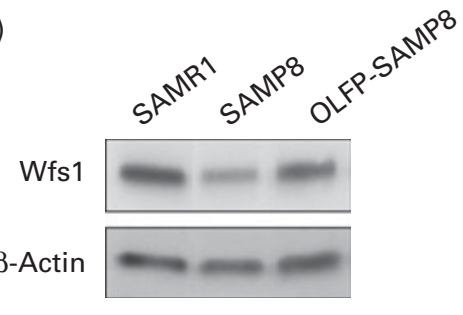

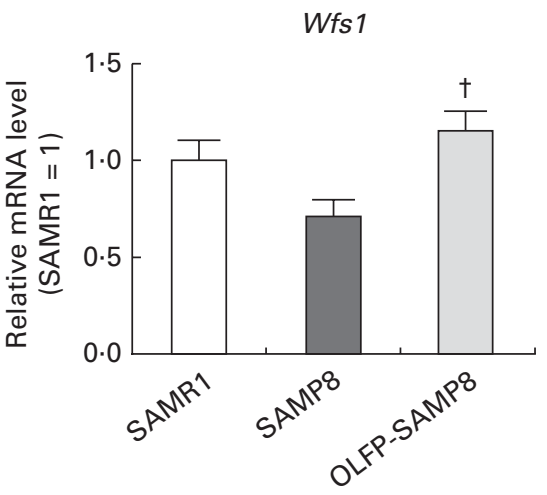

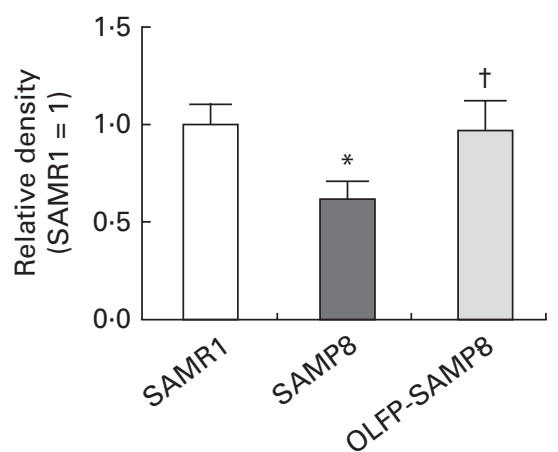

Fig. 2. Effects of oligomerised lychee fruit-derived polyphenol (OLFP) on the expression of cerebellin 4 (Cb/n4) and Wolfram syndrome 1 (Wfs1) in the hippocampus. (a) mRNA expression levels of Cbln4 and Wfs 1 in the hippocampus. Total RNA were extracted from the hippocampus of each mouse and then subjected to quantitative real-time PCR. The expression level of each mRNA was normalised using the 18S ribosomal RNA level. Values are related to an arbitrary unit of senescence-accelerated mouse resistant 1 (SAMR1) mice (set to 1). (b) The expression level of the Wfs1 protein in the hippocampus. Total protein was extracted from the hippocampus of each mouse and then subjected to Western blot analysis. Representative data from the Western blot analysis are shown (left). The expression level of the Wfs1 protein was normalised to that of the $\beta$-actin protein. Values are related to the optical density of SAMR1 mice (set to 1$)$. Values are means ( $n 6$ per group), with their standard errors represented by vertical bars. ${ }^{*}$ Mean values were significantly different from those of SAMR1 mice $(P<0.05)$. † Mean values were significantly different from those of senescence-accelerated mouse prone 8 (SAMP8) mice $(P<0.05)$.

(a)

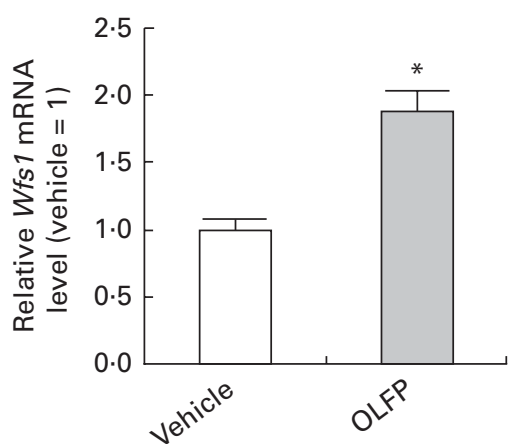

(b)
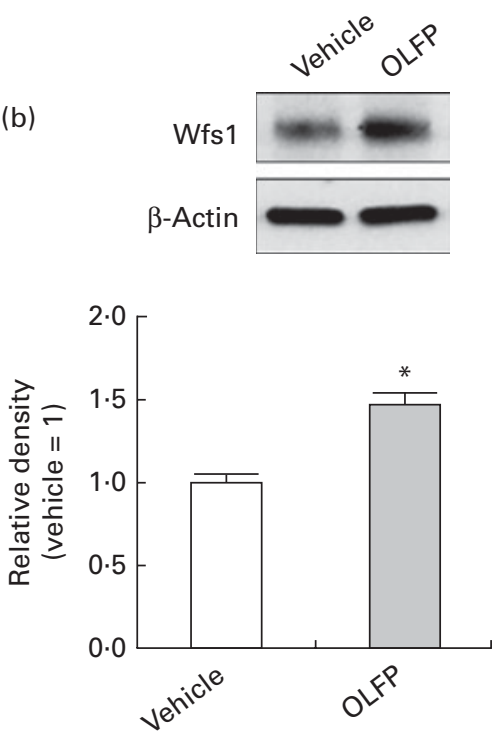

Fig. 3. Expression changes of Wolfram syndrome 1 (Wfs1) in NG108-15 neuronal cells by oligomerised lychee fruit-derived polyphenol (OLFP). Total RNA and protein were extracted from the NG108-15 cells treated with OLFP $(2 \mu \mathrm{g} / \mathrm{ml})$ for 2 and $6 \mathrm{~h}$, respectively. (a) mRNA expression level of Wfs 1 was examined using quantitative real-time PCR, and normalised using the 18S ribosomal RNA level. Values are related to the arbitrary unit of vehicle-treated NG108-15 cells (set to 1). (b) Expression of the Wfs1 protein was examined by Western blot analysis. Representative data from the Western blot analysis are shown (top). Expression level of the Wfs1 protein was normalised to that of the $\beta$-actin protein. Values are related to the optical density of the vehicle-treated cells (set to 1$)$. Values are means, with their standard errors represented by vertical bars, $n 3$. * Mean values were significantly different from those of the vehicle-treated cells $(P<0.05)$. 
(a)
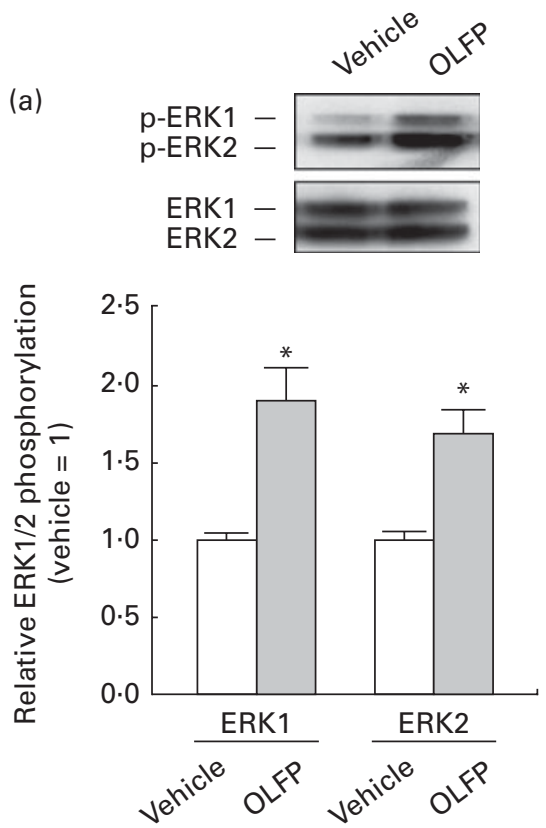

(b)
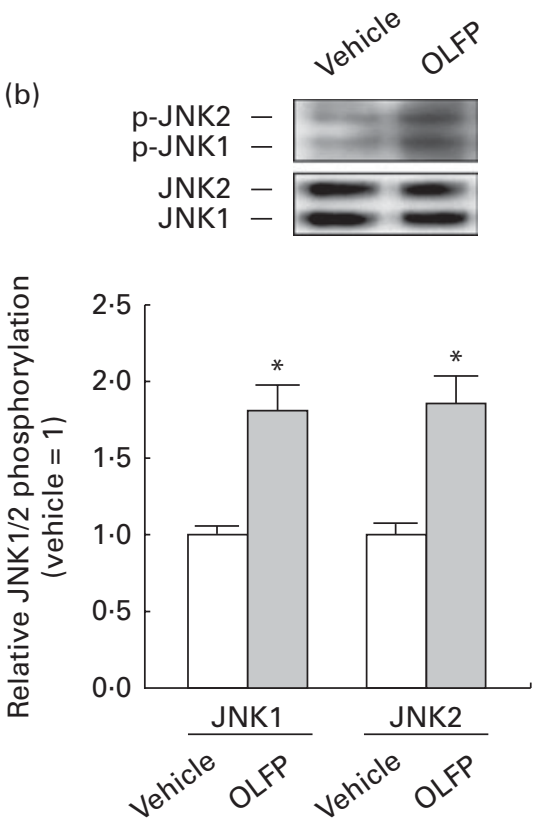

Fig. 4. Phosphorylation of extracellular signal-regulated kinase (p-ERK) and c-Jun N-terminal kinase (p-JNK) in NG108-15 neuronal cells by oligomerised lychee fruit-derived polyphenol (OLFP). Total protein was extracted from the NG108-15 cells treated with OLFP ( $2 \mu \mathrm{g} / \mathrm{ml})$ for $30 \mathrm{~min}$, and subjected to Western blot analysis. Representative data from Western blot analyses are shown (top). Phosphorylation levels of (a) ERK1/2 and (b) JNK1/2 were normalised to the expression levels of ERK1/2 and JNK1/2, respectively. Data were expressed as ratios, with the value of the vehicle-treated cells being set to 1 . Values are means ( $n 3$ ), with their standard errors represented by vertical bars. * Mean values were significantly different from those of the vehicle-treated cells $(P<0.05)$.

(a)

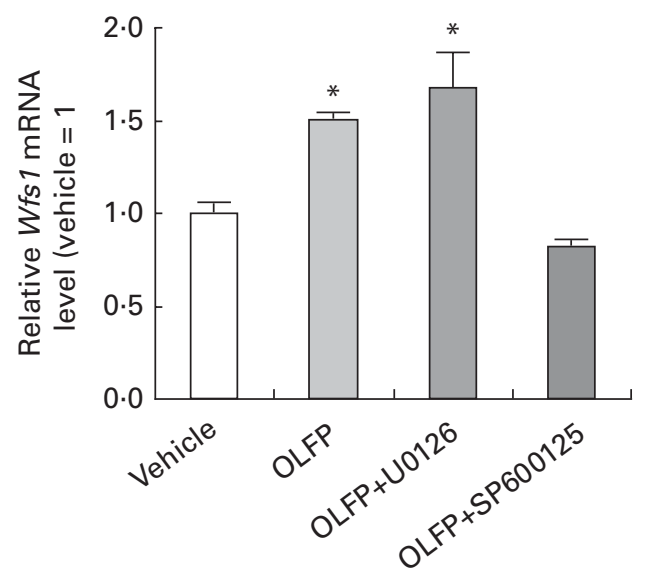

(b)
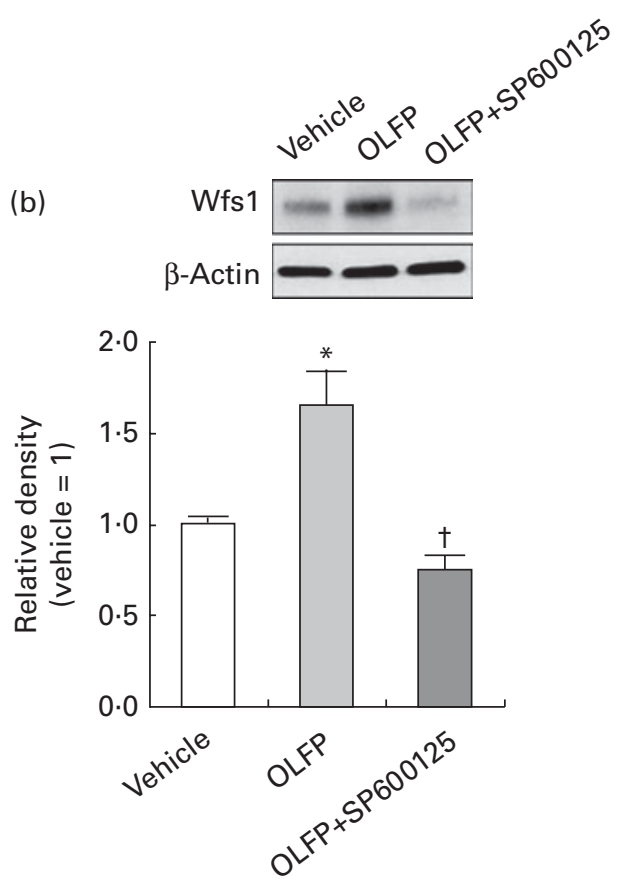

Fig. 5. Effects of mitogen-activated protein kinase inhibitor on the oligomerised lychee fruit-derived polyphenol (OLFP)-induced enhanced expression of Wolfram syndrome 1 (Wfs 1) in neuronal cells. NG108-15 cells were incubated in culture medium with $5 \mu \mathrm{M}-\mathrm{U} 0126$ (an extracellular signal-regulated kinase inhibitor) or $50 \mu \mathrm{M}$-SP600125 (a c-Jun N-terminal kinase inhibitor) for $30 \mathrm{~min}$ before treatment with OLFP, and the expression of Wfs1 was examined. (a) The mRNA expression level of Wfs1 was examined using quantitative real-time PCR, and was normalised using the 18S ribosomal RNA level. Values are related to the arbitrary unit of the vehicle-treated cells (set to 1). (b) Expression of the Wfs1 protein was examined by Western blot analysis. Representative data from the Western blot analysis are shown (top). Expression level of the Wfs1 protein was normalised to that of the $\beta$-actin protein. Values are related to the optical density of the vehicle-treated cells (set to 1). Values are means, with their standard errors represented by vertical bars, $n 3$. ${ }^{*}$ Mean values were significantly different from those of the vehicle-treated cells $(P<0.05)$. † Mean value was significantly different from that of the OLFP-treated cells $(P<0.05)$. 
(a)

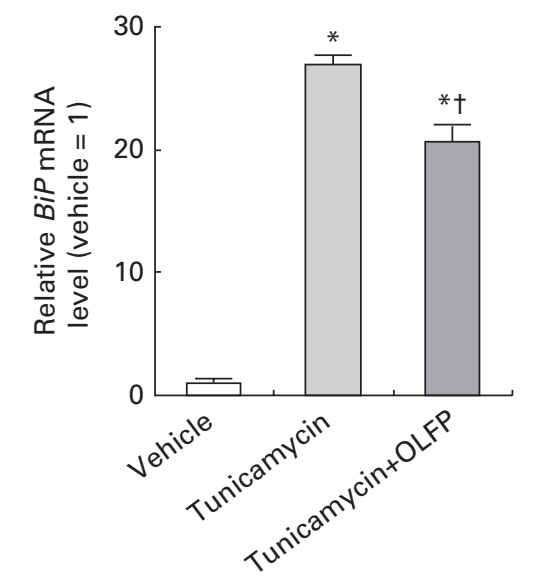

(b)
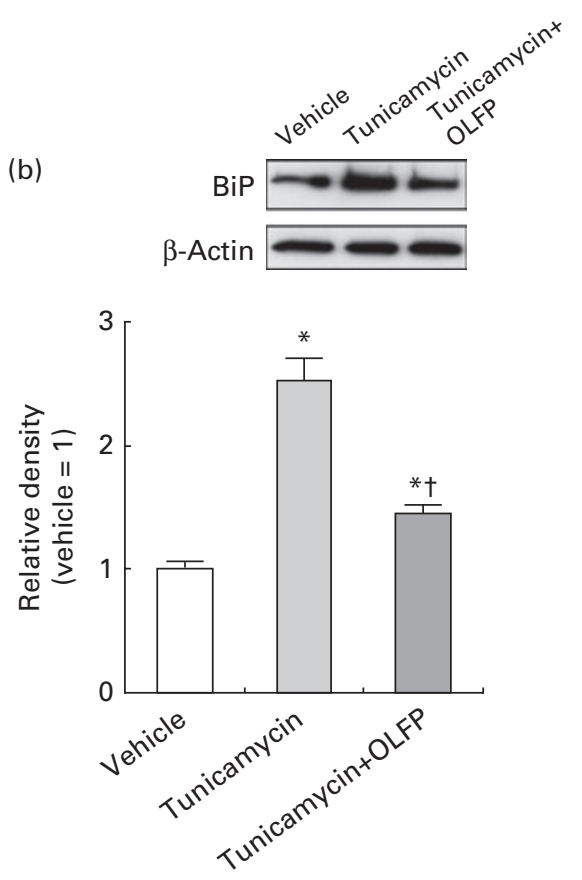

Fig. 6. Effects of oligomerised lychee fruit-derived polyphenol (OLFP) on the expression of the tunicamycin-induced endoplasmic reticulum (ER) stress marker $\mathrm{BiP}$ (immunoglobulin heavy chain-binding protein) in NG108-15 neuronal cells. NG108-15 cells were incubated in culture medium with OLFP for $1 \mathrm{~h}$ before the treatment with the ER stress inducer tunicamycin $(0.5 \mu \mathrm{g} / \mathrm{ml})$ for $12 \mathrm{~h}$, and the expression of BiP was examined. (a) The mRNA expression level of BiP was examined using quantitative real-time PCR, and normalised using the $18 \mathrm{~S}$ ribosomal RNA level. Values are related to the arbitrary unit of the vehicle-treated cells (set to 1). (b) Expression of the BiP protein was examined by Western blot analysis. Representative data from the Western blot analysis are shown (top). Expression level of the BiP protein was normalised to that of the $\beta$-actin protein. Values are related to the optical density of the vehicle-treated cells (set to 1). Values are means $(n 3)$, with their standard errors represented by vertical bars. ${ }^{\star}$ Mean values were significantly different from those of the vehicle-treated cells $(P<0.05)$. $\dagger$ Mean values were significantly different from those of the tunicamycin-treated cells $(P<0.05)$.

and protein expression of BiP was markedly up-regulated by the treatment with the ER stress inducer tunicamycin in NG108-15 cells, but OLFP significantly diminished such tunicamycin-induced BiP expression. These results suggest that OLFP has protective effects against ER stress in neuronal cells.

\section{Discussion}

At present, for most patients with dementia or $\mathrm{AD}$, a full recovery from cognitive impairment is extremely difficult using existing medications and other approaches, such as physical exercise and intake of supplements. Therefore, a reduction in the risk of dementia involving $\mathrm{AD}$, as well as by developing a medication specifically for $\mathrm{AD}$, is thought to be effective methods to avoid an increase in the number of patients with such disorders. The concept of 'MCI' has been proposed as a diagnosis for the risk of developing dementia and $\mathrm{AD}^{(37)}$. $\mathrm{MCI}$ is categorised as an intermediate state of cognitive function between changes seen in normal ageing individuals and those with dementia or $\mathrm{AD}^{(37)}$. Thus, the establishment of risk-reducing strategies for early cognitive impairment, such as MCI, is thought to lead to a reduction in the risk of dementia involving AD. SAMP8 mice are a strain of mice with accelerated senescence, and have recently been the focus of attention, as they show several alterations that have also been described in $\mathrm{AD}$ patients ${ }^{(38)}$. SAMP8 mice overproduce a compound similar to $A \beta$, and $A \beta$ granules in the hippocampus of SAMP8 mice are observed from 6 months of age ${ }^{(17)}$. The formation of $\mathrm{A} \beta$ granules in the hippocampus increases with age. As a result, amyloid plaques occur late in life (approximately 20 months) ${ }^{(18)}$. Therefore, SAMP8 mice used in the present experiment ( 4 months old) are thought to correspond to the MCI state in humans, which is supported by previous experimental results that, when 4-month-old SAMP8 mice were used, spatial learning and memory was not impaired in a Morris water maze test, although memory deficiency was observed when mice were subjected to passive avoidance and conditioned fear memory tests ${ }^{(9,30)}$. In the present study, OLFP intake seemed to have attenuating effects on early cognitive impairment and MCI in SAMP8 mice, which corresponds to MCI in humans - a result that is expected to lead to a risk reduction of AD.

Polyphenols are a large group of powerful antioxidants that are found in fruits and vegetables, such as grapes and lychee fruits, and in beverages including tea and wine. Usually, the bioactivity and absorption coefficients of polyphenols in the body seem to be implicated in their components - the polymerisation degree of polyphenols. For example, Fujii et al. ${ }^{\text {(39) }}$ found that oligomerisation of purified grape seed polyphenols is more conducive to higher absorption rates in vivo than the non-oligomerised form. We have also previously found that antioxidative effects of OLFP on adipocytes are stronger than those of non-OLFP, and that the intensity of 
the antioxidative effects of these polyphenols in adipocytes is clearly reflected in the expression of genes for adipokines ${ }^{(40)}$. Moreover, the inhibitory effects of procyanidins on pancreatic lipase activity increased with a rise in the degree of polymerisation from dimer to pentamer ${ }^{(41)}$. Recently, interesting evidence about the relationship between the degree of polymerisation in polyphenols and its effects on cognitive impairment in an $\mathrm{AD}$ model of amyloid precursor protein transgenic mice has been published. Wang et al. ${ }^{(42)}$ fractionated a grape-derived polyphenolic mixer to monomer and polymer-enriched proanthocyanidin components, and gave each fraction to amyloid precursor protein transgenic mice and rats. As a result, only the monomeric fraction improved the cognitive function of amyloid precursor protein transgenic mice, and metabolites from the monomeric fraction were able to selectively reach and accumulate in the brain of rats. Therefore, OLFP might have the attenuating effects on the memory deficiency of SAMP8 mice due to the presence of catechin-type monomers and oligomers derived from the lychee fruit ${ }^{(20)}$. However, as for an inhibitory effect on angioten$\sin I$ in the conversion of enzyme activities, among procyanidins consisting of dimers, tetramers or hexamers, since procyanidin tetramers have the lowest half-maximal inhibitory concentration value ${ }^{(43)}$, proper polymeric forms of polyphenols are considered to differ according to specific biological effects.

Mechanisms responsible for the polyphenol-induced attenuating effects on cognitive impairment have been found by several research groups. Mitochondrial dysfunction and production of $\mathrm{A} \beta$ in the brains of patients with $\mathrm{AD}$ result in the generation of reactive oxygen species and subsequent oxidative stress in neuronal cells ${ }^{(44,45)}$. Oxidative stress-induced neuronal cell damage in the brain involving the hippocampus is considered to be associated with AD-related cognitive dysfunction $^{(44,45)}$. Dietary supplementation with antioxidant molecules, such as polyphenols, reportedly brings about an improvement in memory deficiency with a reduction in the level of oxidative stress in the hippocampus of old rats and rats injected with $A \beta^{(46,47)}$. Furthermore, the intake of green tea catechin has diminished $A \beta$ through a modification of the enzyme activity of secretase, and has activated the cyclic AMP response element binding protein, which is considered to be necessary in the formation of long-term memory ${ }^{(48)}$, in the hippocampus of a mouse ${ }^{(9,21)}$. In the present study, we performed DNA array analysis to search novel OLFP target molecules in the hippocampus. Consequently, Wfs1 was identified. Moreover, we found that OLFP directly enhanced the expression of $W f S 1$ in neuronal cells via the activation of JNK. Inoue et $a l .^{(49)}$ identified human WFS1 as the gene responsible for the Wolfram syndrome (OMIM 222300), which is a neurodegenerative disorder defined by insulindependent diabetes mellitus and progressive optic atrophy. OLFP could significantly enhance the expression of Wfs 1 in the hippocampus of SAMP8 mice, which appears to be related to the OLFP-induced attenuation of memory deficiency, as shown in conditioned fear memory tests, because abundant expression of the WfS1 transcript has been confirmed in the CA1 region of the rodent hippocampus ${ }^{(34)}$. Moreover, it has been suggested that the CA1 region is implicated in contextual fear conditioning and extinction ${ }^{(50-52)}$. However, it is difficult to draw conclusions since there were no differences between Wfs1-deficient mice and wild types in conditioned fear memory testing ${ }^{(53)}$. There is no denying that the role of Wfs 1 in memory formation in the hippocampus of the adult brain may be different from that during postnatal brain development. Thus, the relationship between Wsf1 and memory function remains unclear, and further studies are required.

Recent interesting results have indicated that the Wfs1 protein localises primarily to the ER membrane, and has protective effects against ER stress ${ }^{(25,27,33)}$. In fact, Wfs 1 deficiency in mice leads to a progressive loss of pancreatic $\beta$-cells, impaired glucose tolerance and cell-cycle progression, accompanied by the activation of ER stress/unfolded protein response pathways ${ }^{(25,27)}$. The accumulation of unfolded proteins following disruptions of proper protein folding and modification in the ER causes ER stress and activates a signalling network called the unfolded protein response ${ }^{(54)}$. When cellular protective changes mediated by the unfolded protein response fail to restore folding capacity, ER stress leads to a maladaptive response and apoptosis ${ }^{(26)}$. Several neurodegenerative diseases including $\mathrm{AD}$ have been suggested to have an accumulation of misfolded proteins, which leads to altered neuronal connectivity, neuronal death and a dysfunctional process of ER-associated degradation machinery ${ }^{(26,55,56)}$, which shows that ER stress is involved in the pathology of $\mathrm{AD}$ and in other neurodegenerative diseases. Indeed, the administration of $A \beta$ induced the activation of ER stress in cultured neurons and in rabbits in vivo ${ }^{(57,58)}$. Also, caspase-12 mediates the apoptosis of ER stress-specific neuronal cells via $A \beta^{(59)}$. OLFP significantly attenuated the tunicamycininduced up-regulation of BiP expression in NG108-15 cells, indicating that OLFP possesses the potential for protective effects against ER stress in neuronal cells in part due to an up-regulation of $W f s 1$. Thus, it is conceivable that the administration of OLFP brings about the risk reduction of AD due to the attenuation of ER stress in the hippocampus. Nevertheless, since no significant differences in the expression levels of the $\mathrm{BiP}$ protein in the hippocampus were observed among the SAMR1, SAMR8 and SAMP8-OLFP mice (data not shown), although the expression level of the Wfs1 protein in the hippocampus of SAMP8 mice was lower than that of SAMR1, and OLFP significantly enhanced the expression of the Wfs1 protein, additional investigations are needed to reveal the relationship between the anti-ER stress effect of OLFP and the risk reduction of $\mathrm{AD}$.

In conclusion, the results obtained from the present study suggest that the administration of OLFP attenuates contextual fear memory deficiency in SAMP8 mice. Moreover, we identified $W f s 1$ as a novel factor, which may relate to the OLFP-induced attenuation of cognitive impairment in the hippocampus.

\section{Acknowledgements}

The present study was partially supported by Grants-in-Aid for Specific Project Research from the Ministry of Education, Culture, Sports, Science and Technology of Japan. T. S., S. S. 
and J. O. collected and analysed the data. T. S., K. K., H. N. and H. F. prepared the materials for the experiment. T. F. and K. A. provided the experimental guidance and contributed to the experimental design. T. K., T. I. and H. O. contributed to the interpretation of the results and gave critical comments. T. S. and H. O. wrote the manuscript. All authors read and approved the final manuscript. There are no conflicts of interest.

\section{References}

1. Ittner LM \& Götz J (2011) Amyloid $\beta$ and tau - a toxic pas de deux in Alzheimer's disease. Nat Rev Neurosci 12, 65-72.

2. Allegri RF, Butman J, Arizaga RL, et al. (2007) Economic impact of dementia in developing countries: an evaluation of costs of Alzheimer type dementia in Argentina. Int Psychogeriatr 19, 705-718.

3. M Prince and J Jackson (editors) (2009) World Alzheimer Report 2009. London: Alzheimer's Disease International.

4. Arts IC \& Hollman PC (2005) Polyphenols and disease risk in epidemiologic studies. Am J Clin Nutr 81, 317S-325S.

5. Scalbert A, Manach C, Morand C, et al. (2005) Dietary polyphenols and the prevention of diseases. Crit Rev Food Sci Nutr 45, 287-306.

6. Ramassamy C (2006) Emerging role of polyphenolic compounds in the treatment of neurodegenerative diseases: a review of their intracellular targets. Eur J Pharmacol 545 , 51-64.

7. Singh M, Arseneault M, Sanderson T, et al. (2008) Challenges for research on polyphenols from foods in Alzheimer's disease: bioavailability, metabolism, and cellular and molecular mechanisms. J Agric Food Chem 56, 4855-4873.

8. Luchsinger JA \& Mayeux R (2004) Dietary factors and Alzheimer's disease. Lancet Neurol 3, 579-587.

9. Li Q, Zhao HF, Zhang ZF, et al. (2009) Long-term green tea catechin administration prevents spatial learning and memory impairment in senescence-accelerated mouse prone- 8 mice by decreasing $A \beta_{1-42}$ oligomers and upregulating synaptic plasticity-related proteins in the hippocampus. Neuroscience 163, 741-749.

10. Rezai-Zadeh K, Arendash GW, Hou H, et al. (2008) Green tea epigallocatechin-3-gallate (EGCG) reduces beta-amyloid mediated cognitive impairment and modulates tau pathology in Alzheimer transgenic mice. Brain Res 1214, 177-187.

11. Wang J, Ferruzzi MG, Ho L, et al. (2012) Brain-targeted proanthocyanidin metabolites for Alzheimer's disease treatment. J Neurosci 32, 5144-5150.

12. Wang J, Ho L, Zhao W, et al. (2008) Grape-derived polyphenolics prevent $\mathrm{A} \beta$ oligomerization and attenuate cognitive deterioration in a mouse model of Alzheimer's disease. J Neurosci 28, 6388-6392.

13. Miyamoto M, Kiyota N, Yamazaki A, et al. (1986) Age related changes in learning and memory in the senescence accelerated mouse (SAM). Physiol Behav 38, 399-406.

14. Takeda T (2009) Senescence accelerated mouse (SAM) with special references to neurodegeneration models, SAMP8 and SAMP10 mice. Neurochem Res 34, 639-659.

15. Flood JF \& Morley JE (1998) Learning and memory in the SAMP8 mouse. Neurosci Biobehav Rev 22, 1-20.

16. Canudas AM, Gutierrez Cuesta J, Rodríguez MI, et al. (2005) Hyperphosphorylation of microtubule associated protein tau in senescence accelerated mouse (SAM). Mech Ageing Dev 126, 1300-1304.
17. Del Valle J, Duran-Vilaregut J, Manich G, et al. (2010) Early amyloid accumulation in the hippocampus of SAMP8 mice. J Alzheimers Dis 19, 1303-1315.

18. Morley JE, Kumar VB, Bernardo AE, et al. (2000) $\beta$-Amyloid precursor polypeptide in SAMP8 mice affects learning and memory. Peptides 21, 1761-1767.

19. Manich G, Mercader C, del Valle J, et al. (2010) Characterization of amyloid- $\beta$ granules in the hippocampus of SAMP8 mice. J Alzheimers Dis 19, 1303-1315.

20. Fujii H, Nishioka H, Wakame K, et al. (2008) Acute, subchronic and genotoxicity studies conducted with Oligonol, an oligomerized polyphenol formulated from lychee and green tea extracts. Food Chem Toxicol 46, 3553-3562.

21. Lee JW, Lee YK, Ban JO, et al. (2009) Green tea (-)-epigallocatechin-3-gallate inhibits $\beta$-amyloid-induced cognitive dysfunction through modification of secretase activity via inhibition of ERK and NF-кB pathways in mice. $J$ Nutr 139, 1987-1993.

22. Vingtdeux V, Giliberto L, Zhao H, et al. (2010) AMP-activated protein kinase signaling activation by resveratrol modulates amyloid- $\beta$ peptide metabolism. $J$ Biol Chem $\mathbf{2 8 5}$, 9100-9013.

23. Ogasawara J, Kitadate K, Nishioka H, et al. (2009) Oligonol, a new lychee fruit-derived low-molecular form of polyphenol, enhances lipolysis in primary rat adipocytes through activation of the ERK1/2 pathway. Phytother Res 23, 1626-1633.

24. Noh JS, Park CH \& Yokozawa T (2011) Treatment with Oligonol, a low-molecular polyphenol derived from lychee fruit, attenuates diabetes-induced hepatic damage through regulation of oxidative stress and lipid metabolism. $\mathrm{Br} \mathrm{J}$ Nutr 106, 1013-1022.

25. Fonseca SG, Ishigaki S, Oslowski CM, et al. (2010) Wolfram syndrome 1 gene negatively regulates ER stress signaling in rodent and human cells. J Clin Invest 120, 744-755.

26. Lindholm D, Wootz H \& Korhonen L (2006) ER stress and neurodegenerative diseases. Cell Death Differ 13, 385-392.

27. Ueda K, Kawano J, Takeda K, et al. (2005) Endoplasmic reticulum stress induces Wfs1 gene expression in pancreatic $\beta$-cells via transcriptional activation. Eur J Endocrinol 153, $167-176$.

28. Pincus D \& Walter P (2012) A first line of defense against ER stress. J Cell Biol 198, 277-279.

29. Fujiwara T, Mishima T, Kofuji T, et al. (2006) Analysis of knock out mice to determine the role of HPC $1 /$ syntaxin $1 \mathrm{~A}$ in expressing synaptic plasticity. $J$ Neurosci $\mathbf{2 6}$, 5767-5776.

30. Ohta A, Akiguchi I, Seriu N, et al. (2001) Deterioration in learning and memory of fear conditioning in response to context in aged SAMP8 mice. Neurobiol Aging 22, 479-484.

31. Cohen J (1988) Statistical Power Analysis for the Behavioral Sciences, 2nd ed. Hillsdale, NJ: Lawrence Erlbaum.

32. Rogan MT \& LeDoux JE (1996) Emotion: systems, cells, synaptic plasticity. Cell $\mathbf{8 5}$, 469-475.

33. Rigoli L, Lombardo F \& Di Bella C (2011) Wolfram syndrome and WFS1 gene. Clin Genet 79, 103-117.

34. Takeda K, Inoue H, Tanizawa Y, et al. (2001) WFS1 (Wolfram syndrome 1) gene product: predominant subcellular localization to endoplasmic reticulum in cultured cells and neuronal expression in rat brain. Hum Mol Genet 10, 477-484.

35. Briviba K, Pan L \& Rechkemmer G (2002) Red wine polyphenols inhibit the growth of colon carcinoma cells and modulate the activation pattern of mitogen-activated protein kinases. J Nutr 132, 2814-2818. 
36. Wu H, Qi H, Iwasaki D, et al. (2009) JNK-dependent NFATc1 pathway positively regulates IL-13 gene expression induced by (-)-epigallocatechin-3-gallate in human basophilic KU812 cells. Free Radic Biol Med 47, 1028-1038.

37. Petersen RC (2011) Clinical practice. Mild cognitive impairment. $N$ Engl J Med 364, 2227-2234.

38. Morley JE, Farr SA, Kumar VB, et al. (2012) The SAMP8 mouse: a model to develop therapeutic interventions for Alzheimer's disease. Curr Pharm Des 18, 1123-1130.

39. Fujii H, Sun B, Nishioka H, et al. (2007) Evaluation of the safety and toxicity of the oligomerized polyphenol Oligonol. Food Chem Toxicol 45, 378-387.

40. Sakurai T, Nishioka H, Fujii H, et al. (2008) Antioxidative effects of a new lychee fruit-derived polyphenol mixture, Oligonol, converted into a low-molecular form in adipocytes. Biosci Biotechnol Biochem 72, 463-476.

41. Sugiyama H, Akazome Y, Shoji T, et al. (2007) Oligomeric procyanidins in apple polyphenol are main active components for inhibition of pancreatic lipase and triglyceride absorption. Agric Food Chem 55, 4604-4609.

42. Wang J, Ferruzzi MG, Ho L, et al. (2012) Brain-targeted proanthocyanidin metabolites for Alzheimer's disease treatment. J Neurosci 325, 144-150.

43. Ottaviani JI, Actis-Goretta L, Villordo JJ, et al. (2006) Procyanidin structure defines the extent and specificity of angiotensin I converting enzyme inhibition. Biochimie $\mathbf{8 8}$, 359-365.

44. Ferreiro E, Baldeiras I, Ferreira IL, et al. (2012) Mitochondrialand endoplasmic reticulum-associated oxidative stress in Alzheimer's disease: from pathogenesis to biomarkers. Int J Cell Biol 2012, 735206.

45. Massaad CA (2011) Neuronal and vascular oxidative stress in Alzheimer's disease. Curr Neuropharmacol 9, 662-673.

46. Haque AM, Hashimoto M, Katakura M, et al. (2006) Longterm administration of green tea catechins improves spatial cognition learning ability in rats. J Nutr 136, 1043-1047.

47. Haque AM, Hashimoto M, Katakura M, et al. (2008) Green tea catechins prevent cognitive deficits caused by $\mathrm{A} \beta 1-40$ in rats. J Nutr Biochem 19, 619-626.
48. Sakamoto K, Karelina K \& Obrietan K (2011) CREB: a multifaceted regulator of neuronal plasticity and protection. J Neurochem 116, 1-9.

49. Inoue $\mathrm{H}$, Tanizawa $\mathrm{Y}$, Wasson $\mathrm{J}$, et al. (1998) A gene encoding a transmembrane protein is mutated in patients with diabetes mellitus and optic atrophy (Wolfram syndrome). Nat Genet 20, 143-148.

50. Matsuo N, Reijmers L \& Mayford M (2008) Spine-typespecific recruitment of newly synthesized AMPA receptors with learning. Science 319, 1104-1107.

51. Radulovic J, Kammermeier J \& Spiess J (1998) Relationship between fos production and classical fear conditioning: effects of novelty, latent inhibition, and unconditioned stimulus preexposure. J Neurosci 18, 7452-7461.

52. Tronson NC, Schrick C, Guzman YF, et al. (2009) Segregated populations of hippocampal principal CA1 neurons mediating conditioning and extinction of contextual fear. $J$ Neurosci 29, 3387-3394.

53. Luuk H, Plaas M, Raud S, et al. (2008) Wfs1-deficient mice display impaired behavioural adaptation in stressful environment. Behav Brain Res 198, 334-345.

54. Lai E, Teodoro T \& Volchuk A (2007) Endoplasmic reticulum stress: signaling the unfolded protein response. Physiology 22, 193-201.

55. Bence NF, Sampat RM \& Kopito RR (2001) Impairment of the ubiquitin-proteasome system by protein aggregation. Science 292, 1552-1555.

56. Soto C (2003) Unfolding the role of protein misfolding in neurodegenerative diseases. Nat Rev Neurosci 4, 49-60.

57. Ferreiro E, Resende R, Costa R, et al. (2006) An endoplasmicreticulum-specific apoptotic pathway is involved in prion and amyloid- $\beta$ peptides neurotoxicity. Neurobiol Dis $\mathbf{2 3}$, 669-678.

58. Ghribi O, Herman MM, DeWitt DA, et al. (2001) A $\beta$ (1-42) and aluminum induce stress in the endoplasmic reticulum in rabbit hippocampus, involving nuclear translocation of gadd 153 and NF-кB. Brain Res Mol Brain Res 96, 30-38.

59. Nakagawa T, Zhu H, Morishima N, et al. (2000) Caspase-12 mediates endoplasmic-reticulum-specific apoptosis and cytotoxicity by amyloid- $\beta$. Nature $\mathbf{4 0 3}, 98-103$. 